TAIWANESE JOURNAL OF MATHEMATICS

Vol. 14, No. 4, pp. 1411-1416, August 2010

This paper is available online at http://www.tjm.nsysu.edu.tw/

\title{
ON THE WEAK ANALOGUE OF THE TROTTER-KATO THEOREM
}

\author{
Tanja Eisner and András Serény
}

\begin{abstract}
In the Trotter-Kato approximation theorem for $C_{0}$-semigroups on Banach spaces, we replace the strong by the weak operator topology and discuss the validity of the relevant implications.
\end{abstract}

\section{INTRODUCTION}

Trotter-Kato approximation theorems relate the convergence of a sequence of $C_{0^{-}}$ semigroups $\left(T_{n}(t)\right)_{t \geq 0}$ on a Banach space to the convergence of the corresponding generators $A_{n}$ and their resolvents $R\left(\lambda, A_{n}\right)$, in each case for the strong operator topology. They are still an interesting topic in semigroup theory (see, e.g., Bobrowski [3]) and are used, e.g., for partial and stochastic (partial) differential equations and in numerical analysis (see, e.g., Ito and Kappel [6]).

In this note we consider the following question: Do these results still hold for convergence with respect to the weak operator topology?

First positive results have been proved by Yosida [9, Section IX.12] for semigroups on locally convex spaces assuming equicontinuity. Later, using the concept of bi-continuous semigroups introduced by Kuhnemund [7], Albanese, Kuhnemund [1] and Albanese, Mangino [2] extended the Trotter-Kato theorems to this situation and applied it to Markov semigroups. They also needed equicontinuity, an assumption far too strong for the weak operator topology.

In this paper we discuss this situation and prove or disprove the implications in the following Trotter-Kato theorem quoted from Engel, Nagel [5, Theorem III.4.8] if we replace the strong by the weak operator topology.

Received May 7, 2008, accepted September 23, 2008.

Communicated by Sen-Yen Shaw.

2000 Mathematics Subject Classification: 47D06, 34K07.

Key words and phrases: Trotter-Kato theorem, Approximation, Weak operator topology.

The second author was supported by the DAAD-PPP-Hungary Grant, project number D/05/01422. 
Theorem 1.1. (First Trotter-Kato theorem). Let $T(\cdot)$ and $T_{n}(\cdot), n \in \mathbb{N}$, be $C_{0}$ semigroups on a Banach space $X$ with generators $A$ and $A_{n}, n \in \mathbb{N}$, respectively. Assume that for some constants $M \geq 1, \omega \in \mathbb{R}$ the estimates $\|T(t)\|,\left\|T_{n}(t)\right\| \leq$ $M e^{\omega t}$ hold for all $t \geq 0$ and $n \in \mathbb{N}$. Let further $D$ be a core for $A$. Consider the following assertions.

(a) $D \subset D\left(A_{n}\right)$ for every $n \in \mathbb{N}$ and $\lim _{n \rightarrow \infty} A_{n} x=A x$ for all $x \in D$.

(b) For every $x \in D$ there exists $x_{n} \in D\left(A_{n}\right)$ such that $\lim _{n \rightarrow \infty} x_{n}=x$ and $\lim _{n \rightarrow \infty} A_{n} x_{n}=A x$.

(c) $\lim _{n \rightarrow \infty} R\left(\lambda, A_{n}\right) x=R(\lambda, A) x$ for every $x \in X$ and some $\lambda$ with $\operatorname{Re} \lambda>\omega$.

(d) $\lim _{n \rightarrow \infty} T_{n}(t) x=T(t) x$ for every $x \in X$, uniformly in $t$ on compact intervals.

Then $(a) \Rightarrow(b) \Leftrightarrow(c) \Leftrightarrow(d)$, while (b) does not imply $(a)$.

We will prove that $(d) \Rightarrow(c) \Rightarrow(b) \Leftarrow(a)$ while none of conditions $(a)$, $(b)$ and $(c)$ implies $(d)$ for the weak operator topology (not even for bounded generators). This immediately shows that also the corresponding implications in the second Trotter-Kato theorem [5, Theorem III.4.9] fail for the weak operator topology. Moreover, for this topology neither $(a)$ nor $(b)$ implies $(c)$ in general. We finish the note with an open question.

\section{The Results}

We first give an example of bounded generators $A$ and $A_{n}, n \in \mathbb{N}$, generating contraction semigroups such that $\lim _{n \rightarrow \infty} A_{n} x=A x$ weakly for every $x \in X$, but the semigroups $\left(e^{t A_{n}}\right)$ do not converge weakly to $\left(e^{t A}\right)$. This shows that the implication $(a) \Rightarrow(d)$ (as well as $(b) \Rightarrow(d)$ ) in Theorem 1.1 does not hold for the weak operator topology.

Example 2.1. Consider $X:=l^{p}, 1 \leq p<\infty$, and the operators $\widetilde{\mathrm{A}}_{n}$ defined by

$$
\begin{aligned}
& \tilde{\mathrm{A}}_{n}\left(x_{1}, \ldots, x_{n}, x_{n+1}, \ldots, x_{2 n}, \ldots\right) \\
& :=\left(x_{n+1}, x_{n+2}, \ldots, x_{2 n}, x_{1}, x_{2}, \ldots, x_{n}, x_{2 n+1}, x_{2 n+2}, \ldots\right)
\end{aligned}
$$

exchanging the first $n$ coordinates of a vector with its next $n$ coordinates. Then $\left\|\tilde{\mathrm{A}}_{n}\right\|=1$ implies that $\tilde{\mathrm{A}}_{n}$ generates a $C_{0}$-semigroup $\tilde{\mathrm{T}}_{n}(\cdot)$ satisfying $\left\|\widetilde{\mathrm{T}}_{n}(t)\right\| \leq e^{t}$ for every $n \in \mathbb{N}$ and $t \geq 0$.

The operators $\tilde{\mathrm{A}}_{n}$ converge weakly (but not strongly) to zero as $n \rightarrow \infty$. Moreover, $\widetilde{\mathrm{A}}_{n}^{2}=I$ for every $n \in \mathbb{N}$. Therefore 


$$
\begin{aligned}
\widetilde{\mathrm{T}}_{n}(t) & =\sum_{k=0}^{\infty} \frac{t^{k} \tilde{\mathrm{A}}_{n}^{k}}{k !}=\sum_{k=0}^{\infty} \frac{t^{2 k+1}}{(2 k+1) !} \tilde{\mathrm{A}}_{n}+\sum_{k=0}^{\infty} \frac{t^{2 k}}{(2 k) !} I \\
& =\frac{e^{t}-e^{-t}}{2} \tilde{\mathrm{A}}_{n}+\frac{e^{t}+e^{-t}}{2} I \stackrel{\sigma}{\longrightarrow} \frac{e^{t}+e^{-t}}{2} I \neq I,
\end{aligned}
$$

where " $\sigma$ " denotes the weak operator topology, and for all $x \in l^{p}$ and $y \in\left(l^{p}\right)^{*}$ the convergence of $\left\{\left\langle\tilde{T}_{n}(t) x, y\right\rangle\right\}$ is uniform on compact time intervals. However, the limit does not satisfy the semigroup law.

By rescaling $A_{n}:=\tilde{\mathrm{A}}_{n}-I$ we obtain a sequence of contractive semigroups converging weakly and uniformly on compact time intervals to a family which is not a semigroup while the bounded generators converge weakly to $-I$ (which is a generator).

Remark 2.2. The above example shows in particular that (in general) the space of all contractive $C_{0}$-semigroups on a Banach space is not complete for the topology corresponding to the weak operator convergence, uniform on compact time intervals. Note that for the strong operator topology this space is complete, a fact essentially used in Eisner, Serény [4].

Next we give an example showing that the implication $(c) \Rightarrow(d)$ does not hold for the weak operator topology. Note that the converse implication (i.e. $(d) \Rightarrow(c)$ ) is true by the representation $R\left(\lambda, A_{n}\right)=\int_{0}^{\infty} e^{-\lambda t} T_{n}(t) d t$ and Lebesgue's theorem. Note further that the implications $(a) \Rightarrow(b)$ and $(c) \Rightarrow(b)$ also remain true for the weak operator topology (the first is trivial and the proof of the second is the same as in the strong case).

Example 2.3. On $X:=l^{2}$ consider the operators $V_{n}:=\left(1-\frac{1}{n}\right) \tilde{\mathrm{A}}_{n}$ for $n \in \mathbb{N}$, where $\tilde{\mathrm{A}}_{n}$ are the operators from the above example. Then $\left\|V_{n}\right\| \stackrel{n}{=} 1-\frac{1}{n}<1$ and hence $1 \notin \sigma\left(V_{n}\right)$. By Foias-Sz.-Nagy [8, Thm. III.8.1] every $V_{n}$ is the cogenerator of a contractive $C_{0}$-semigroup $S_{n}(\cdot)$ on $X$. Recall that the generator $B_{n}$ of the semigroup $S_{n}(\cdot)$ is given by the (negative) Cayley transform of $V_{n}$, i.e., $B_{n}=$ $\left(I+V_{n}\right)\left(I-V_{n}\right)^{-1}$. In our case, all $B_{n}$ are bounded operators as well.

We see that $R\left(1, B_{n}\right)=\frac{1}{2}\left[I-V_{n}^{-1}\right]=\frac{1}{2}\left[I-\left(1-\frac{1}{n}\right)^{-1} \tilde{\mathrm{A}}_{n}\right] \stackrel{\sigma}{\longrightarrow} \frac{I}{2}=$ $R(1,-I)$ as $n \rightarrow \infty$, where we used the identity $\tilde{\mathrm{A}}_{n}=\tilde{\mathrm{A}}_{n}^{-1}$. Assume that the semigroups $S_{n}(\cdot)$ converge weakly and uniformly on compact intervals to the semigroup $\left(e^{-t} I\right)$ generated by $-I$. Then

$$
R^{2}\left(1, B_{n}\right)=-\left.\frac{d}{d \lambda} R\left(\lambda, B_{n}\right)\right|_{\lambda=1}=\int_{0}^{\infty} e^{-t} t S_{n}(t) d t \stackrel{\sigma}{\longrightarrow} R^{2}(1,-I) \text { as } n \rightarrow \infty .
$$

However, since $\tilde{A}_{n}^{2}=I$ for every $n \in \mathbb{N}$ we obtain 


$$
R^{2}\left(1, B_{n}\right)=\frac{1}{4}\left[I-2 V_{n}^{-1}+\left(1-\frac{1}{n}\right)^{-2} I\right] \stackrel{\sigma}{\longrightarrow} \frac{I}{2} \neq R^{2}(1,-I)
$$

which is a contradiction.

We now summarise the results shown above.

Theorem 2.4. Let $T(\cdot)$ and $T_{n}(\cdot), n \in \mathbb{N}$, be $C_{0}$-semigroups on a Banach space $X$ with generators $A$ and $A_{n}, n \in \mathbb{N}$, respectively. Assume that for some constants $M \geq 1, \omega \in \mathbb{R}$ the estimates $\|T(t)\|,\left\|T_{n}(t)\right\| \leq M e^{\omega t}$ hold for all $t \geq 0$ and $n \in \mathbb{N}$. Let further $D$ be a core for A. Consider the following assertions.

(a) $D \subset D\left(A_{n}\right)$ for every $n \in \mathbb{N}$ and $\lim _{n \rightarrow \infty} A_{n} x=A x$ weakly for all $x \in D$.

(b) For every $x \in D$ there exists $x_{n} \in D\left(A_{n}\right)$ such that $\lim _{n \rightarrow \infty} x_{n}=x$ weakly and $\lim _{n \rightarrow \infty} A_{n} x_{n}=A x$ weakly.

(c) $\lim _{n \rightarrow \infty} R\left(\lambda, A_{n}\right) x=R(\lambda, A) x$ weakly for every $x \in X$ and some $\lambda$ with $\operatorname{Re} \lambda>\omega$.

(d) $\lim _{n \rightarrow \infty} T_{n}(t) x=T(t) x$ weakly for every $x \in X$, uniformly in $t$ on compact intervals.

Then $(d) \Rightarrow(c) \Rightarrow(b) \Leftarrow(a)$, while none of conditions $(a)$, $(b)$ and $(c)$ implies (d) in general (even if all operators $A_{n}$ are bounded).

Remark 2.5. Example 2.1 shows also that none of the conditions $(a)$ and $(b)$ implies $(c)$, even if all operators are bounded. Indeed, for $\lambda$ with $|\lambda|>1$ we have

$$
\begin{aligned}
R\left(\lambda, \tilde{\mathrm{A}}_{n}\right) & =\sum_{k=0}^{\infty} \frac{\tilde{\mathrm{A}}_{n}^{k}}{\lambda^{k+1}}=\sum_{k=0}^{\infty} \frac{1}{\lambda^{2 k+2}} \tilde{\mathrm{A}}_{n}+\sum_{k=0}^{\infty} \frac{1}{\lambda^{2 k+1}} I \\
& =\frac{1}{\lambda^{2}-1} \tilde{\mathrm{A}}_{n}+\frac{\lambda}{\lambda^{2}-1} I \stackrel{\sigma}{\longrightarrow} \frac{\lambda}{\lambda^{2}-1} I \neq R(\lambda, 0) .
\end{aligned}
$$

Moreover, by analyticity of $R\left(\cdot, \tilde{\mathrm{A}}_{n}\right)$ on $\rho\left(\tilde{\mathrm{A}}_{n}\right)=\mathbb{C} \backslash\{-1,1\}$, or by direct calculation, the representation $R\left(\lambda, \tilde{\mathrm{A}}_{n}\right)=\frac{1}{\lambda^{2}-1} \tilde{\mathrm{A}}_{n}+\frac{\lambda}{\lambda^{2}-1} I$ holds for every $\lambda \neq \pm 1$ and every $n \in \mathbb{N}$, so property $(c)$ in Theorem 2.4 fails even for all $\lambda \in \mathbb{C}$.

Remark 2.6. Let $X$ be a Banach space, $T_{n}(\cdot), n \in \mathbb{N}$, and $T(\cdot)$ be $C_{0^{-}}$ semigroups with generators $A_{n}, n \in \mathbb{N}$, and $A$, respectively, satisfying $\|T(t)\|$, $\left\|T_{n}(t)\right\| \leq M e^{\omega t}$ for some contants $M \geq 1, \omega \in \mathbb{R}$ and all $t \geq 0$ and $n \in \mathbb{N}$, and take $\lambda$ with $\operatorname{Re} \lambda>\omega$. If $\lim _{n \rightarrow \infty} T_{n}(t)=T(t)$ weakly and uniformly on compact 
time intervals, then by the representation $R^{k}(\lambda, A)=\frac{1}{(k-1) !} \int_{0}^{\infty} e^{-\lambda t} t^{k-1} T_{n}(t) d t$ we have

$$
\lim _{n \rightarrow \infty} R^{k}\left(\lambda, A_{n}\right)=R^{k}(\lambda, A) \text { weakly for all } k \in \mathbb{N} .
$$

Note that property (1) for one $\lambda$ implies the same property for all $\lambda$ with $\operatorname{Re} \lambda>\omega$ and the convergence in (1) is uniform for $\lambda$ in compact sets of the corresponding open right halfplane. This follows from the power series representation for the resolvent, a standard connectedness argument and Cauchy's formula for the derivatives. Further, by the same reason (1) is equivalent to

$$
\begin{aligned}
& \lim _{n \rightarrow \infty} R\left(\lambda, A_{n}\right) \\
= & R(\lambda, A) \text { weakly and uniformly on compact sets in }\{\lambda: \operatorname{Re} \lambda>\omega\} .
\end{aligned}
$$

As we saw in Example 2.3, property (2) is not equivalent to weak convergence of the resolvents for some (fixed) $\lambda$ as is the case for strong operator convergence.

Therefore the following question appears naturally.

Question 2.7. Is property (1) for somelall $\lambda$ equivalent to weak convergence of the corresponding semigroups uniform on compact time intervals?

Remark 2.8. If all generators $A_{n}$ are bounded and satisfy $\left\|A_{n}\right\| \leq M$ for some constant $M \geq 0$ and all $n \in \mathbb{N}$, then the answer on the above question is "yes". This follows immediately from the Dunford representation $T_{n}(t)=$ $\frac{1}{2 \pi i} \int_{\{|z|=M+1\}} e^{t z} R\left(z, A_{n}\right) d z$ and (2).

\section{ACKNOWLEDGMENTS}

The authors are very thankful to Rainer Nagel, the editor Sen-Yen Shaw and the referee for very helpful comments.

\section{REFERENCES}

1. A. Albanese and F. Kühnemund, Trotter-Kato approximation theorems for locally equicontinuous semigroups, Riv. Mat. Univ. Parma, 7 (2002), 19-53.

2. A. Albanese and E. Mangino, Trotter-Kato theorems for bi-continuous semigroups and applications to Feller semigroups, J. Math. Anal. Appl., 289 (2004), 477-492.

3. A. Bobrowski, On limitations and insufficiency of the Trotter-Kato theorem, Semigroup Forum, 75 (2007), 317-336. 
4. T. Eisner and A. Sereny, Category theorems for stable $C_{0}$-semigroups, Ergodic Theory Dynamical Systems, 29 (2009), 487-494.

5. K.-J. Engel and R. Nagel, One-parameter Semigroups for Linear Evolution Equations, Graduate Texts in Mathematics, Vol. 194, Springer-Verlag, New York, 2000.

6. K. Ito and F. Kappel, The Trotter-Kato theorem and approximation of PDEs, Math. Comp., 67 (1998), 21-44.

7. F. Kühnemund, A Hille-Yosida theorem for bi-continuous semigroups, Semigroup Forum, 67 (2003), 205-225.

8. B. Sz.-Nagy and C. Foiaş, Harmonic Analysis of Operators on Hilbert Space, NorthHolland Publ. Comp, Akadémiai Kiado, Amsterdam, Budapest, 1970.

9. K. Yosida, Functional Analysis, fourth edition. Die Grundlehren der mathematischen Wissenschaften, Springer-Verlag, New York-Heidelberg, 1974.

\title{
Tanja Eisner
}

Mathematisches Institut,

Universităt Tubingen

Auf der Morgenstelle 10

D-70176, Tübingen

Germany

E-mail: talo@fa.uni-tuebingen.de

\author{
András Serény \\ Department of Mathematics and its Applications \\ Central European University \\ Nádor utca 9 \\ H-1051 Budapest \\ Hungary \\ E-mail: sandris@elte.hu
}

